\title{
Nonlinear Waves in Rods and Beams of Power-Law Materials
}

\author{
Dongming Wei, ${ }^{1}$ Piotr Skrzypacz, ${ }^{1}$ and Xijun $Y_{u^{2}}$ \\ ${ }^{1}$ Department of Mathematics, School of Science and Technology, Nazarbayev University, Astana 010000, Kazakhstan \\ ${ }^{2}$ Institute of Applied Physics and Computational Mathematics, P.O. Box 8009, Beijing 100088, China \\ Correspondence should be addressed to Dongming Wei; dongming.wei@nu.edu.kz
}

Received 16 March 2017; Revised 4 May 2017; Accepted 4 June 2017; Published 13 July 2017

Academic Editor: Xin-Lin Gao

Copyright (C) 2017 Dongming Wei et al. This is an open access article distributed under the Creative Commons Attribution License, which permits unrestricted use, distribution, and reproduction in any medium, provided the original work is properly cited.

Some novel traveling waves and special solutions to the 1D nonlinear dynamic equations of rod and beam of power-law materials are found in closed forms. The traveling solutions represent waves of high elevation that propagates without change of forms in time. These waves resemble the usual kink waves except that they do not possess bounded elevations. The special solutions satisfying certain boundary and initial conditions are presented to demonstrate the nonlinear behavior of the materials. This note demonstrates the apparent distinctions between linear elastic and nonlinear plastic waves.

\section{Introduction}

Free vibrations of rods and beams of power-law materials are considered. Analytic traveling wave solutions to the wave equations for power-law materials (see $[1,2])$ are obtained which represent kink waves of single elevation that propagates without change of forms in time. It is shown that, unlike the wave equations for linear materials, the nonlinear wave equations do not allow arbitrary traveling wave forms in an infinite rod or beam. The results demonstrate that the traveling fronts of the waves may sharpen or flatten as the wave speeds increase depending upon the power-law index $n$ and the bulk modulus. For $n>1$, the wave fronts sharpen, whereas for $0<n<1$, the fronts flatten as the wave speeds increase. The solutions also demonstrate that the speeds of the nonlinear traveling waves depend not only on the material properties but also on the initial energy-level. It is well known that the speeds of waves for the linear elastic materials $(n=$ 1, Hooke's law) depend only on the material properties in contrast to that of the waves in nonlinear materials. As far as we know these solutions are not available in literature, even though there are numerous research papers and books devoted to the discovery and study of traveling waves in elastic and plastic solids (see [3-7] for details). In the case of rods and beams of finite length, we also present some special solutions satisfying certain boundary and initial conditions. The closed formula solutions are expressed in terms of nonEuclidean sine functions (cf. [8]), which differ from the
Euclidean sine functions corresponding to the waves in rods and beams of linear elastic materials.

The note is organized as follows. In Section 2, the powerlaw constitutive stress-strain equation is introduced. In Section 3, the potential energy and derivations of the wave equations of power-law materials are outlined. In Sections 4 and 5, closed-form solutions are derived. And, finally the results are summarized in Section 6.

\section{Hollomon's Equation}

It is well known that, in uniaxial state, the following powerlaw stress and strain relation is used for certain elastoplastic materials:

$$
\sigma=K|\varepsilon|^{n-1} \varepsilon, \quad 0<n<\infty,
$$

where $\sigma$ is the axial stress, $\varepsilon$ is the axial strain, and $K$ and $n$ are engineering constants with values depending on the specific material. The materials satisfying (1) sometimes are also referred to as Ludwick or as Hollomon's materials in literature (cf. [1,2]). Many heat-treated metals are well-known power-law materials. For a given annealed metal or alloy, $K$ and $n$ depend on the heat treatment received by the metal or alloy. The values of $n$ are typically between 0 and 1 for such metals. For a comprehensive list of experimental values of $K$ and $n$ of common annealed industrial metals, see, for example, [9]. For some geological materials, such as certain rocks or ice, however, the values of $n$ are greater than 1 . In 
some biological tissues, experiments also indicate that the power-law index $n$ satisfies $0<n<1$ for bones such as tibia and femur, while $n>1$ for cartilages such as common carotid artery and abdominal aorta (see, e.g., [10, 11]). For a given value of $0<n<1$, the stress-strain curve defined by (1) can result in a rapid increase in the yield stress for small strains or strain hardening. However, it can be the opposite for values of $n>1$, for which large strains produce small stress or softening. For these reasons, $n$ is called the strain-hardening or strain-softening exponent. Study of the mechanical properties of these heat-treated metals is very important in industries (see, e.g., [12], for stress analysis of beam columns made of Ludwick materials). If we allow $n=1$, then (1) reduces to Hooke's law for linear elastic material and the constant $K$, also called the bulk modulus, equals the corresponding Young's modulus E. Power-law materials are a special case of a more general class of materials called Hencky plastics [13]. Physically, the constitutive equation (1) describes the hardening or softening of materials showing an elastic-plastic transition. In the following, bold letters are used to denote vectors or matrices. A vector is considered as a single row matrix. The transpose of a matrix $\mathbf{A}$ is denoted by $\mathbf{A}^{\tau}$, and the inner product of two vectors $\mathbf{u}$ and $\mathbf{v}$ by $\mathbf{u v}^{\tau}$. The time derivative $\partial \mathbf{u} / \partial t$ is denoted by $\dot{\mathbf{u}}$. Let $\mathbf{u}(x, y, z, t)=(u(x, y, z, t), v(x, y, z, t), w(x, y, z, t))$ denote the displacement vector,

$$
\begin{aligned}
\varepsilon_{x} & =\frac{\partial u}{\partial x} \\
\varepsilon_{y} & =\frac{\partial v}{\partial y}, \\
\varepsilon_{z} & =\frac{\partial w}{\partial z} \\
\gamma_{x y} & =\frac{1}{2}\left(\frac{\partial u}{\partial y}+\frac{\partial v}{\partial x}\right), \\
\gamma_{y z} & =\frac{1}{2}\left(\frac{\partial v}{\partial z}+\frac{\partial w}{\partial y}\right), \\
\gamma_{z x} & =\frac{1}{2}\left(\frac{\partial w}{\partial x}+\frac{\partial u}{\partial z}\right)
\end{aligned}
$$

the strain components, and $\sigma_{x}, \sigma_{y}, \sigma_{z}, \tau_{x y}, \tau_{y z}$, and $\tau_{z x}$ the corresponding stress components. The following generalized power law can be derived from the Hencky total deformation theory [13]:

$$
\begin{aligned}
& \left\{\begin{array}{c}
\sigma_{x} \\
\sigma_{y} \\
\sigma_{z} \\
\tau_{x y} \\
\tau_{y z} \\
\tau_{z x}
\end{array}\right\}=\frac{K\|D(\mathbf{u})\|^{n-1}}{(1+\nu)(1-2 v)} \\
& \left(\begin{array}{cccccc}
1-v & v & v & 0 & 0 & 0 \\
v & 1-v & v & 0 & 0 & 0 \\
v & v & 1-v & 0 & 0 & 0 \\
0 & 0 & 0 & \frac{1-2 \nu}{2} & 0 & 0 \\
0 & 0 & 0 & 0 & \frac{1-2 \nu}{2} & 0 \\
0 & 0 & 0 & 0 & 0 & \frac{1-2 \nu}{2}
\end{array}\right)\left\{\begin{array}{c}
\varepsilon_{x} \\
\varepsilon_{y} \\
\varepsilon_{z} \\
\gamma_{x y} \\
\gamma_{y z} \\
\gamma_{z x}
\end{array}\right\},
\end{aligned}
$$

where $\|D(\mathbf{u})\|=\sqrt{\varepsilon_{x}^{2}+\varepsilon_{y}^{2}++\varepsilon_{z}^{2}+2 \gamma_{x y}^{2}+2 \gamma_{y z}^{2}+2 \gamma_{z x}^{2}}$, where $n, K$, and $v$ are the material constants; see also Wei [14]. Note that (3) is the three-dimensional version of (1). In the following two sections, wave equations of bars and beams made of the power-law elastoplastic materials are derived by (3) and the assumption of the Euler-Bernoulli beam theory. There are similar versions of generalized powerlaw stress-strain relations for strain-hardening or strainsoftening material in the literature and similar wave equations can be derived (see, e.g., [15-20]).

\section{The Nonlinear Wave Equations}

The potential energy for a power-law elastoplastic body occupying a three-dimension body $V$ can by defined by

$$
U=\frac{1}{n+1} \int_{V} \sigma \varepsilon^{\tau} d V
$$

where $\boldsymbol{\varepsilon}=\left(\varepsilon_{x}, \varepsilon_{y}, \varepsilon_{z}, \gamma_{x y}, \gamma_{x z}, \gamma_{y z}\right)$ and $\boldsymbol{\sigma}=\left(\sigma_{\mathbf{x}}, \sigma_{\mathbf{y}}, \sigma_{\mathrm{z}}, \tau_{\mathbf{x y}}\right.$, $\left.\tau_{\mathrm{xz}}, \tau_{\mathrm{yz}}\right)$. The Lagrangian energy functional $I(\mathbf{u})$ equals the kinetic energy $T$ minus the elastoplastic potential energy $U$ plus the work $W$ done by external force. It can be written as

$$
\begin{aligned}
I(\mathbf{u})= & \frac{1}{2} \int_{V} \rho \dot{\mathbf{u}} \dot{\mathbf{u}}^{\tau} d V-\frac{1}{n+1} \int_{V} \sigma \varepsilon^{\tau} d V+\int_{V} \mathbf{f} \mathbf{u}^{\tau} d V \\
& +\int_{\partial V} \mathbf{t u}^{\tau} d S,
\end{aligned}
$$

where $\rho$ is the density, $\dot{\mathbf{u}}=(\dot{u}, \dot{v}, \dot{w})$ the velocity, $\mathbf{f}=$ $\left(f_{x}, f_{y}, f_{z}\right)$ the body force, and $\mathbf{t}=\left(t_{x}, t_{y}, t_{z}\right)$ the surface force. See, for example, [21], for a standard definition of $I(\mathbf{u})$. For a uniaxial bar of infinite length with cross-sectional area $A(x)$, subject to axial force and zero surface force, we have $\boldsymbol{\sigma}=\left(\sigma_{x}, 0,0,0,0,0\right), \mathbf{u}=(u(x, t), 0,0), \sigma_{x}=K\left|\varepsilon_{x}\right|^{n-1} \varepsilon_{x}$, $\mathbf{f}=(f(x, t), 0,0)$, and $\mathbf{t}=(0,0,0)$. For an Euler beam of infinite length, it is assumed that the components of the displacement satisfy $u(x, y, t)=-y(\partial v / \partial x), v=v(x, t)$, $w=0, \mathbf{f}=(0, r(x, t), 0)$, and $\mathbf{t}=(0,0,0)$. Therefore $\varepsilon_{x}=$ $\partial u / \partial x=-y\left(\partial^{2} v / \partial x^{2}\right), \varepsilon_{x y}=(1 / 2)(\partial u / \partial y+\partial v / \partial x)=0$, and $\varepsilon_{y}=\varepsilon_{x z}=\varepsilon_{y z}=\varepsilon_{z}=0$. The potential energies for the bar and the beam are given by

$$
\begin{aligned}
& U=\frac{1}{n+1} \int_{-\infty}^{+\infty} K A\left|\frac{\partial u}{\partial x}\right|^{n+1} d x \\
& U=\frac{1}{n+1} \int_{-\infty}^{+\infty} K I_{n}\left|\frac{\partial^{2} v}{\partial x^{2}}\right|^{n+1} d x
\end{aligned}
$$

respectively, where $I_{n}=\int_{A}|y|^{n+1} d y d z$ is the generalized second moment of inertia of the beam. The $x$-axis is taken to be the axial direction of the bar and the beam. For rods and beams of finite length $L$, the corresponding Lagrangian functions are given by replacing $-\infty$ and $+\infty$ in (6) and (7) by 0 and $L$, respectively. Note that the assumptions made in this section on the elastoplastic bars and the beams are standard assumptions frequently made for elastic bars and beams (see, e.g., [22, 23], for details). The corresponding linear wave 
equations of elastic bars and beams corresponding to $n=1$ have been studied extensively.

For completeness, the derivation of the wave equations of the power-law materials given in [14] is outlined here. It is well known that Hamilton's principle seeks an equilibrium state in time dependent mechanical systems (see, e.g., [21]).

Specifically, Hamilton's principle requires that we seek a displacement $\mathbf{u}$ so that, for any time interval $\left[t_{1}, t_{2}\right], \mathbf{u}\left(t_{1}\right)=$ $\mathbf{u}\left(t_{2}\right)$ and $\dot{\mathbf{u}}\left(t_{1}\right)=\dot{\mathbf{u}}\left(t_{2}\right)$, and for all displacement of the form $\mathbf{u}+\tau \mathbf{v}$, where $\tau$ is any real number, the first variation of the energy functional $I$ satisfies

$$
\delta I=\left.\int_{t_{1}}^{t_{2}} \frac{d}{d \tau}[I(\mathbf{u}(t)+\tau \mathbf{v}(t))]\right|_{\tau=0} d t=0
$$

for all $\mathbf{v}$ satisfying $\mathbf{v}\left(t_{1}\right)=\mathbf{v}\left(t_{2}\right)=\mathbf{0}$ and $\dot{\mathbf{v}}\left(t_{1}\right)=\dot{\mathbf{v}}\left(t_{2}\right)=\mathbf{0}$. The combination $\mathbf{u}(t)+\tau \mathbf{v}(t)$ is referred to as an admissible displacement for the mechanical system since it is required to satisfy some boundary conditions. It can be shown that if the displacement $\mathbf{u}$ satisfies (8) of Hamilton's principle, then it must also satisfy a differential wave equation under certain conditions. In particular, suppose that the cross-sectional area, denoted by $A$, is a nonzero constant, and then for the rod, we have

$$
\rho \frac{\partial^{2} u}{\partial t^{2}}=K \frac{\partial}{\partial x}\left(\left|\frac{\partial u}{\partial x}\right|^{n-1} \frac{\partial u}{\partial x}\right)+f, \quad x \in \mathbb{R}, t \in \mathbb{R}^{+}
$$

and for the corresponding Euler beam

$$
\rho A \frac{\partial^{2} v}{\partial t^{2}}=-\frac{\partial^{2}}{\partial x^{2}}\left(K I_{n}\left|\frac{\partial^{2} v}{\partial x^{2}}\right|^{n-1} \frac{\partial^{2} v}{\partial x^{2}}\right)+A r
$$

$$
x \in \mathbb{R}, t \in \mathbb{R}^{+} .
$$

When $n=1$, (9) reduces to the standard wave equation for the elastic bar

$$
\rho \frac{\partial^{2} u}{\partial t^{2}}=K \frac{\partial^{2} u}{\partial x^{2}}+f, \quad x \in \mathbb{R}, t \in \mathbb{R}^{+}
$$

and (10) to the standard wave equation for the elastic Euler beam

$$
\rho A \frac{\partial^{2} v}{\partial t^{2}}=-\frac{\partial^{2}}{\partial x^{2}}\left(K I \frac{\partial^{2} v}{\partial x^{2}}\right)+A r, \quad x \in \mathbb{R}, t \in \mathbb{R}^{+} .
$$

The quantity $I_{n}$ reduces to the second moment of inertia, $I_{n}=\int_{A}|y|^{n+1} d A$ reduces to $I$ when $n=1$ in the elastic beam theory, and the material constant $K$ becomes Young's modulus $E$ for linear elastic materials. In deriving the wave equations (9) and (10), we have made the assumption that the solutions $u$ and $v$ are continuously differentiable and their appropriate lower order derivatives are bounded or vanishing when $|x| \rightarrow \infty$. By (8), we get

$$
\begin{aligned}
& \int_{t_{1}}^{t_{2}} \int_{-\infty}^{+\infty}\left(\rho A \dot{u} \dot{v}-K A\left|\frac{\partial u}{\partial x}\right|^{n-1} \frac{\partial u}{\partial x} \frac{\partial v}{\partial x}+A f v\right) d x d t \\
& \quad=0
\end{aligned}
$$

Using integration by parts and interchange of the order of integration, with $v\left(t_{1}\right)=v\left(t_{2}\right)=0$, and assuming that $\lim _{x \rightarrow \pm \infty}|\partial u(x, t) / \partial x|^{n-1}(\partial u(x, t) / \partial x)$ is bounded by a constant independent of $t$ and $\lim _{x \rightarrow \pm \infty} v(x, t)=0$ uniformly in $t$, we get the following:

$$
\begin{aligned}
& \int_{t_{1}}^{t_{2}} \int_{-\infty}^{+\infty}\left(-\rho A \ddot{u}+\frac{\partial}{\partial x}\left(K A\left|\frac{\partial u}{\partial x}\right|^{n-1} \frac{\partial u}{\partial x}\right)+A f\right) \\
& \cdot v d x d t=0
\end{aligned}
$$

from (13). Since $v, t_{1}$, and $t_{2}$ are arbitrary and $A \neq 0$, we then get (9) from (14). The corresponding beam equation (10) can be derived similarly which was reported in [14].

\section{Traveling Waves in Rods and Beams of Arbitrary Length}

In the following we will derive some traveling wave solutions to (9) and (10) for $0<n<\infty$ and $n \neq 1$. As far as we know, these solutions are not available in literature, even though there are numerous research papers and books devoted to the discovery and study of traveling waves in elastic and plastic solids. For the study of traveling waves in nonlinear beam equations based on Hooke's law $(n=1)$ for elastic materials, see, for example, [3-5]. Also, see [6, 7, 24, 25], for more results of traveling waves in solids. Assuming that $f=r=0$ in (9) and (10), we have

$$
\frac{\partial^{2} u}{\partial t^{2}}=c^{2} \frac{\partial}{\partial x}\left(\left|\frac{\partial u}{\partial x}\right|^{n-1} \frac{\partial u}{\partial x}\right), \quad x \in \mathbb{R}, t \in \mathbb{R}^{+}
$$

for the bar and

$$
\frac{\partial^{2} v}{\partial t^{2}}=\widehat{c}^{2} \frac{\partial^{2}}{\partial x^{2}}\left(\left|\frac{\partial^{2} v}{\partial x^{2}}\right|^{n-1} \frac{\partial^{2} v}{\partial x^{2}}\right), \quad x \in \mathbb{R}, t \in \mathbb{R}^{+}
$$

for the beam, where $c^{2}=K / \rho$ and $\hat{c}^{2}=-K I_{n} / \rho A$, respectively. We look for traveling wave solutions of the form $g(x-\lambda t)$ for both (15) and (16), where $\lambda$ denotes a constant and $g$ is a function to be determined. Let $\phi(t)=|t|^{n-1} t$, where $n$ is the index in power-law (3). The inverse of $\phi$ is $\phi^{-1}(t)=|t|^{(1-n) / n} t$ since

$$
\begin{aligned}
\left(\phi \circ \phi^{-1}\right)(t) & =\left.\left.|| t\right|^{(1-n) / n} t\right|^{n-1}|t|^{(1-n) / n} t \\
& =|t|^{(n-1) / n}|t|^{(1-n) / n} t=t .
\end{aligned}
$$

First, let $\xi=x-\lambda t$ and substitute $u(x, t)=g(\xi)$ into (15), so $\lambda^{2} g^{\prime \prime}=c^{2}\left(\phi\left(g^{\prime}\right)\right)^{\prime}$. After integration we get $\lambda^{2} g^{\prime}=$ $c^{2} \phi\left(g^{\prime}\right)+c_{1}$, where $c_{1}$ is an arbitrary constant. Suppose that $\lim _{x \rightarrow \infty}(\partial u / \partial x)(x, 0)=A$. Since $u(x, 0)=g(x)$ and $g^{\prime}(x)=$ $(\partial u / \partial x)(x, 0)$, we have $c_{1}=\lambda^{2} A-c^{2} \phi(A)$. Looking for nontrivial solutions for $n \neq 1$ and assuming that $A=$ $(c / \lambda)^{2 /(1-n)}$, we get $c_{1}=0$ and $g^{\prime}= \pm(c / \lambda)^{2 /(1-n)}$ which gives the following traveling wave solutions:

$$
u(x, t)= \pm\left(\frac{c}{\lambda}\right)^{2 /(1-n)}(x-\lambda t)+c_{2}
$$


for the bar equation (15). Note that solution (18) includes some physically meaningful solutions. For example, let us consider a semi-infinite bar with initial displacement

$$
u(x, 0)= \begin{cases}\left(\frac{c}{\lambda}\right)^{2 /(1-n)} x & \text { if } 0<x<+\infty \\ 0 & \text { if }-\infty<x \leq 0\end{cases}
$$

and initial velocity

$$
\dot{u}(x, 0)= \begin{cases}-\lambda\left(\frac{c}{\lambda}\right)^{2 /(1-n)} & \text { if } 0<x<+\infty \\ 0 & \text { if }-\infty<x \leq 0\end{cases}
$$

and boundary condition $\lim _{x \rightarrow+\infty}(\partial u / \partial x)(x, t)=(c / \lambda)^{2 /(1-n)}$. A particular solution satisfying these conditions is given by

$$
\begin{aligned}
& u(x, t) \\
& \quad= \begin{cases}\left(\frac{c}{\lambda}\right)^{2 /(1-n)}(x-\lambda t) & \text { if } 0<x-\lambda t<+\infty \\
0 & \text { if }-\infty<x-\lambda t \leq 0\end{cases}
\end{aligned}
$$

which is obtained from (18). The physical interpretation of the initial condition (19) is that half of the bar is initially subject to a constant stress and the other half is free of stress and fixed in position, and the second initial condition (20) means that the bar is initially moving at a constant speed and half of it is instantaneously stopped. Solution (21) explains that if a prestressed semi-infinite axial power-law rod subject to initial conditions $\sigma(x, 0)=K(c / \lambda)^{2 n /(1-n)}$ and $\dot{u}(x, 0)>0$ and boundary conditions $u(0, t)=0$ for $x=0$ and lower order derivatives are bounded or vanishing when $|x| \rightarrow \infty$, then the displacement in the interval $[0, x]$ will be zero at time $t=\left[x^{1+n}|\dot{u}(x, 0)|^{1-n} / c^{2}\right]^{1 /(1+n)}=x / \lambda$ and the restoration of the deformed bar in interval $[0, x(t)]$ to its undeformed configuration has a moving boundary $x(t)$ which is expanding like a kink wave at a velocity $\lambda=$ $c(K / \sigma(x, 0))^{(1-n) / 2 n}$.

In the more general situation, for any value of $c_{1}$, the equation $P(t)=\lambda^{2} t-c^{2} \phi(t)-c_{1}$ has at least one solution since it is continuous, $\lim _{t \rightarrow+\infty} P(t)=+\infty$ and $\lim _{t \rightarrow-\infty} P(t)=-\infty$. Let $P\left(\varepsilon_{0}\right)=0$, and then $g^{\prime}=\varepsilon_{0}$ satisfies $\lambda^{2} g^{\prime}=c^{2} \phi\left(g^{\prime}\right)+c_{1}$. We have the following similar solutions:

$$
u(x, t)= \begin{cases}\varepsilon_{0}(x-\lambda t) & \text { if } 0<x-\lambda t<+\infty \\ 0 & \text { if }-\infty<x-\lambda t \leq 0\end{cases}
$$

satisfying the initial and boundary conditions

$$
\begin{aligned}
& u(x, 0)= \begin{cases}\varepsilon_{0} x & \text { if } 0<x<+\infty \\
0 & \text { if }-\infty<x \leq 0,\end{cases} \\
& \dot{u}(x, 0)= \begin{cases}-\lambda \varepsilon_{0} & \text { if } 0<x<+\infty \\
0 & \text { if }-\infty<x \leq 0\end{cases}
\end{aligned}
$$

for $\lim _{x \rightarrow+\infty}(\partial u / \partial x)(x, t)=\varepsilon_{0}$ and $\lambda \varepsilon_{0}^{2}=c^{2} \phi\left(\varepsilon_{0}\right)+c_{1}$.
For the linear elastic bar, $n=1, \phi\left(g^{\prime}\right)=g^{\prime}$, and if $c_{1}=0$, the equation $\lambda^{2} g^{\prime}=c^{2} \phi\left(g^{\prime}\right)$ is satisfied for any $g^{\prime}$ and also makes $\lambda=c$. This shows that the linear elastic bar equation allows arbitrary traveling wave forms $g$ in $u(x, t)=g(x-\lambda t)$, and however the wave can travel only at a fixed velocity $\lambda=C$. If $c_{1} \neq 0$, then equation $\lambda^{2} g^{\prime}=c^{2} \phi\left(g^{\prime}\right)+c_{1}$ gives $g^{\prime}=\varepsilon_{0}=$ $\left(\lambda^{2}-c^{2}\right) / c_{1}$ and the corresponding solution is (22), which is similar to the solutions for $n \neq 1$.

The above shows that the difference between the nonlinear solution $(n \neq 1)$ and the linear case $(n=1)$ is that all the nonlinear traveling waves have the same shape and the traveling velocity depends not only on the material property but also on the initial stress-level while the linear traveling waves can take any form while keeping a fixed traveling velocity $c$ that depends only on the material property.

Similarly, by substituting $v(x, t)=g(x-\lambda t)$ into the beam equation (16), we get $\lambda^{2} g^{\prime \prime}=\widehat{c}^{2}\left(\phi\left(g^{\prime \prime}\right)\right)^{\prime \prime}$. After integration twice, we get $\lambda^{2} g=\widehat{c}^{2} \phi\left(g^{\prime \prime}\right)+c_{1} \xi+c_{2}$, which gives $\lambda^{2} g=$ $\widehat{c}^{2} \phi\left(g^{\prime \prime}\right)$ by setting $c_{1}=c_{2}=0$. Let $w=g^{\prime}$; we get $w(d w / d g)=$ $g^{\prime \prime}$ and $\lambda^{2} g=\widehat{c}^{2} \phi(w(d w / d g))$. From the last equation, we get

$$
w d w=-\phi^{-1}\left(\frac{\lambda^{2} g}{|\widehat{c}|^{2}}\right) d g=-\left|\frac{\lambda}{\widehat{c}}\right|^{2 / n}|g|^{(1-n) / n} g d g
$$

which gives $\left(g^{\prime}\right)^{2}=C-(2 n /(1+n))|\lambda / \widehat{c}|^{2 / n}|g|^{(1+n) / n}$, where $C$ is the integration constant.

We assume $g^{\prime}(0)=0$ and $g(0)>0$. So, the traveling wave solutions for the corresponding elastoplastic Euler beam are given implicitly

$$
\begin{aligned}
& x-\lambda t \\
& = \pm \int_{0}^{g(x-\lambda t)} \frac{d s}{\sqrt{2|\lambda / \widehat{c}|^{2 / n}(n /(n+1))\left(|g(0)|^{1 / n} g(0)-|s|^{1 / n} s\right)}}
\end{aligned}
$$

which results in the following formula in terms of generalized trigonometric function defined in [26]

$$
g(x-\lambda t)=g(0) \sin _{2,1+1 / n}(B(x-\lambda t)),
$$

where $B=|\widehat{c}|^{1 / n} /|g(0)|^{(n+1) / 2 n} g(0)|\lambda|^{1 / n} \sqrt{2 n /(n+1)}$. Notice that for $n=1$ we obtain the well-known Euclidean sine traveling wave solution for the elastic Euler beam equation. We observe that the amplitude of the wave is determined by the initial condition $v(0,0)$. The traveling waves for elastoplastic beams can be applied to study piezoelectric robots; see [27].

\section{Special Waves in Rods and Beams of Finite Length}

Let us consider some special waves in rods and beams of finite length $L$ with fixed ends. Equations (15) and (16) are solved for $x \in(0, L)$ and $t \in R^{+}$with homogeneous Dirichlet boundary conditions $u(0, t)=u(L, t)=0$ and special initial conditions. We present some special solutions by using the generalized trigonometric functions developed by Drábek and Manásevich [26]. By using the separation of variables 


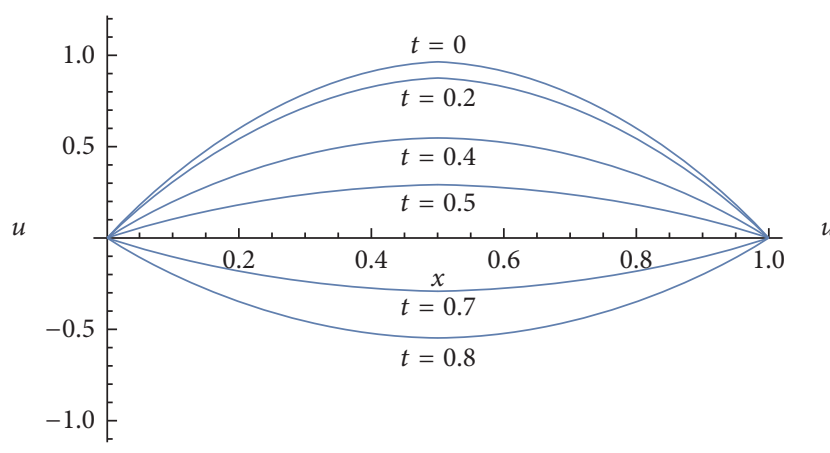

(a)

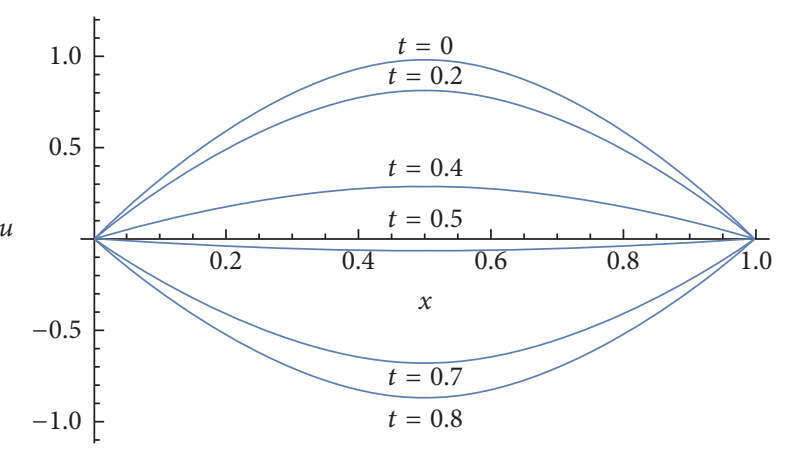

(b)

FiguRE 1: Vibrating power-law strings: $n=0.2$ (a) and $n=0.5$ (b) at $t=0,0.2,0.4,0.5,0.7,0.8$.

$u(x, t)=X(x) T(t)$ in (15) and using boundary conditions $u(0, t)=u(L, t)=0$, we have

$$
\begin{gathered}
\left(\left|X^{\prime}\right|^{n-1} X^{\prime}\right)^{\prime}=\lambda X, \\
\ddot{T}-\lambda c^{2}|T|^{n-1} T=0 .
\end{gathered}
$$

From the first equation and the boundary conditions, we get

$$
\begin{aligned}
\left(\left|X^{\prime}\right|^{n-1} X^{\prime}\right)^{\prime} & =\lambda X, \\
X(0) & =X(L)=0 .
\end{aligned}
$$

By Thm 3.1 in [26], a sequence of solutions to the nonlinear eigenvalue problem (28) are given by $X_{k}=\sin _{n+1,2}\left(k\left(x \pi_{n+1,2} /\right.\right.$ $L)$ ), where $\lambda_{k}=-((k+1) k / 2)\left(\pi_{n+1,2} / L\right)^{n+1}$ and $\pi_{n+1,2}=\int_{0}^{1}(1-$ $\left.t^{2}\right)^{-1 /(n+1)} d t=B(1-1 /(n+1), 1 / 2)$. Let us consider the initial conditions $u(x, 0)=X_{1}(x)$ and $u_{t}(x, 0)=0$. In this case we have $T(0)=1$ and we can solve the second equation in (27). A special solution of this initial value problem is given by

$$
\begin{aligned}
& u(x, t)=\sin _{n+1,2}\left(\frac{x \pi_{n+1,2}}{L}\right) \\
& \cdot \sin _{2, n+1}\left(-\sqrt{2 c}\left(\frac{\pi_{n+1,2}}{L}\right)^{(n+1) / 2} t+\frac{\pi_{2, n+1}}{2}\right) .
\end{aligned}
$$

The time evolution of the special solutions for $c=1, L=1$, $n=0.2$, and $n=0.5$ is presented in Figure 1, respectively.

Similarly, by using the separation of variables $u(x, t)=$ $X(x) T(t)$ in (16), we have

$$
\begin{gathered}
\left(\left|X^{\prime \prime}\right|^{n-1} X^{\prime \prime}\right)^{\prime \prime}=\lambda X, \\
\ddot{T}-\lambda c^{2}|T|^{n-1} T=0 .
\end{gathered}
$$

From the first equation and the boundary condition, we get

$$
\begin{aligned}
\left(\left|X^{\prime \prime}\right|^{n-1} X^{\prime \prime}\right)^{\prime \prime} & =\lambda X, \\
X(0) & =X^{\prime}(0)=X(L)=X^{\prime}(L)=0 .
\end{aligned}
$$

An analytic solution to (31) is not available and is an open problem. This is a nonlinear and nonhomogeneous eigenvalue problem which belongs to an active area of research beyond the scope of this paper, and we post it here as an open problem. Since superposition principle can not be applied to nonlinear problems, the solutions to (15) and (16) with general initial and boundary conditions require further investigations.

\section{Conclusions}

Two nonlinear wave equations are derived: one is for the longitudinal vibrations of a power-law bar and the other is for vertical vibrations of the power-law Euler beam. Analytic traveling wave solutions are found for these two equations for free vibrations in terms of generalized sine functions of two parameters. We recovered the linear elastic waves as special cases of our solutions. The traditional ways of determining the vibrations of a structure made of untreated metals do not apply to the structures of heat-treated metals with hardening and softening mechanical properties. The obtained results can be useful in engineering applications of the power-law materials, such as heat-treated metals and polyimide plastics. Further study of wave propagation and vibrations in structures made of the power-law nonlinear bars and beams seems necessary.

\section{Conflicts of Interest}

The authors declare that there are no conflicts of interest regarding the publication of this paper.

\section{References}

[1] K. Lee, "Large deflections of cantilever beams of non-linear elastic material under a combined loading," International Journal of Non-Linear Mechanics, vol. 37, no. 3, pp. 439-443, 2001.

[2] G. Lewis and F. Monasa, "Large deflections of cantilever beams of non-linear materials of the Ludwick type subjected to an end moment," International Journal of Non-Linear Mechanics, vol. 17, no. 1, pp. 1-6, 1982.

[3] S. S. Antman, Nonlinear Problems of Elasticity, Springer-Verlag, New York, NY, USA, 1995. 
[4] A. R. Champneys, P. J. McKenna, and P. A. Zegeling, "Solitary waves in nonlinear beam equations: stability, fission and fusion," Nonlinear Dynamics, vol. 21, no. 1, pp. 31-53, 2000.

[5] A. V. Porubov and M. G. Velarde, "Dispersive-dissipative solitons in nonlinear solids," Wave Motion, vol. 31, no. 3, pp. 197207, 2000.

[6] W.-S. Duan and J.-B. Zhao, "Solitary waves in a quartic nonlinear elastic bar," Chaos, Solitons and Fractals, vol. 11, no. 8, pp. 1265-1267, 2000.

[7] V. I. Erofeev and N. V. Klyueva, "Solitons and nonlinear periodic strain waves in rods, plates, and shells (a review)," Acoustical Physics, vol. 48, no. 6, pp. 725-741, 2002.

[8] D. Wei, Y. Liu, and M. B. Elgindi, "Some generalized trigonometric sine functions and their applications," Applied Mathematical Sciences, vol. 6, no. 121-124, pp. 6053-6068, 2012.

[9] J. F. Shackelford, in Introduction to Materials Science for Engineers, Prentice-Hall, Englewood Cliffs, NJ, USA, 5th edition, 2000.

[10] Y. C. Fung, Biomechanics: Mechanical Properties of Living Tissue, Springer-Valag, New York, NY, USA, 2nd edition, 1993.

[11] A. J. Grodzinsky, R. D. Kamm, and D. A. Lauffenburger, "Quantitative aspects of tissue engineering: basic issues in kinetics, transport and mechanics," in Principles of Tissue Engineering, R. Lanza, R. Langer, and W. Chick, Eds., R.G. Landes Co, Austin, Tex, USA, 1997.

[12] X. Teng and T. Wierzbicki, "Crush response of an inclined beam-column," Thin-Walled Structures, vol. 41, no. 12, pp. 11291158, 2003.

[13] H. Hencky, "Zur Theorie plastischer Deformationen und der hierdurch im Material hervorgerufenen Nachspannungen," Journal of Applied Mathematics and Mechanics, vol. 4, no. 4, pp. 323-334, 1924.

[14] D. Wei, "Nonlinear wave equations arising in modeling of some power-law elasto-plastic structures," in Proceedings of ICCP6 and CCP, pp. 248-251, Beijing, China, 2005.

[15] C. Atkinson and C. Y. Chen, "On interface dislocations between dissimilar materials with non-linear stress-strain laws (plane strain)," International Journal of Engineering Science, vol. 37, no. 5, pp. 553-573, 1999.

[16] J. Y. Chen, Y. Huang, K. C. Hwang, and Z. C. Xia, "Plane-stress deformation in strain gradient plasticity," Journal of Applied Mechanics, Transactions ASME, vol. 67, no. 1, pp. 105-111, 2000.

[17] C. H. Chou, J. Pan, and S. C. Tang, "Nonproportional loading effects on elastic-plastic behavior based on stress resultants for thin plates of strain hardening materials," International Journal of Plasticity, vol. 10, no. 4, pp. 327-346, 1994.

[18] X.-L. Gao, "A mathematical analysis of the elasto-plastic plane stress problem of a power-law material," IMA Journal of Applied Mathematics, vol. 60, no. 2, pp. 139-149, 1998.

[19] A. E. Giannakopoulos, “Total deformation, plane-strain contact analysis of macroscopically homogeneous, compositionally graded materials with constant power-law strain hardening," Journal of Applied Mechanics, Transactions ASME, vol. 64, no. 4, pp. 853-860, 1997.

[20] Z. K. Wang, X. X. Wei, and X. L. Gao, "A general analytical solution of a strain-hardening elastoplastic plate containing a circular hole subject to biaxial loading-with applications in pressure vessels," International Journal of Pressure Vessels and Piping, vol. 47, no. 1, pp. 35-55, 1991.

[21] M. Petyt, Introduction to Finite Element Vibration Analysis, Cambridge University Press, Cambridge, UK, 1990.
[22] A. C. Ugural, Stresses in Plates and Shells, McGraw-Hill, New York, NY, USA, 1981.

[23] S. Timoshenko, D. H. Young, and W. Weaver Jr., Vibration Problems in Engineering, John Wiley \& Sons, Hoboken, NJ, USA, 1974.

[24] J. Lamb, Elements of Soliton Theory, John Wiley \& Sons, Inc, New York, NY, USA, 1982.

[25] M. Remoissenet, Waves Called Solitons-Concepts and Experiments, Springer, Berlin, Germany, 2nd edition, 1996.

[26] P. Drábek and R. Manásevich, "On the closed solution to some nonhomogeneous eigenvalue problems with $p$-Laplacian," Differential and Integral Equations, vol. 12, no. 6, pp. 773-788, 1999.

[27] H. Hariri, Y. Bernard, and A. Razek, "A traveling wave piezoelectric beam robot," Smart Materials and Structures, vol. 23, no. 2, Article ID 025013, 2014. 


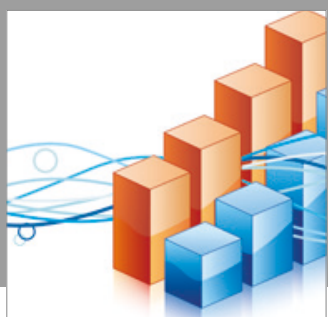

Advances in

Operations Research

vatersals

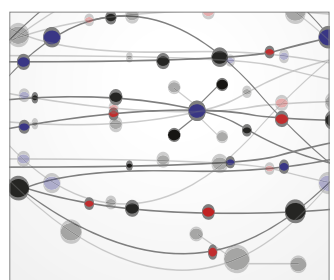

\section{The Scientific} World Journal
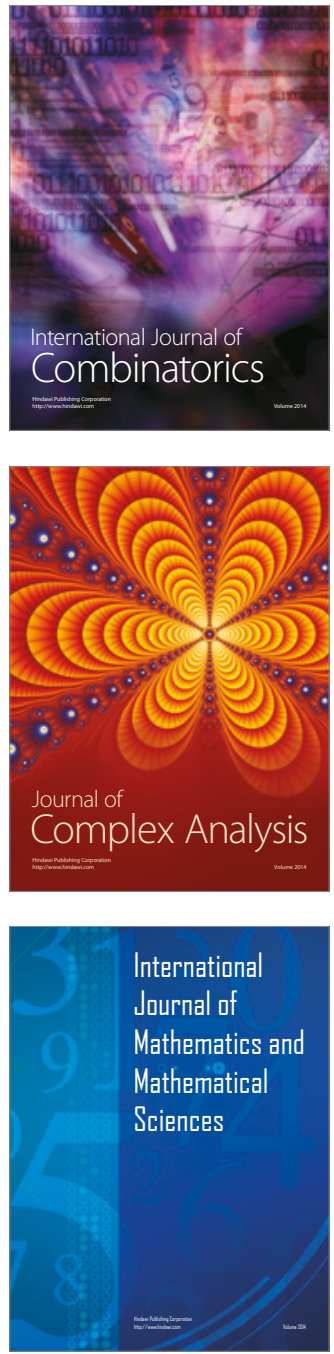
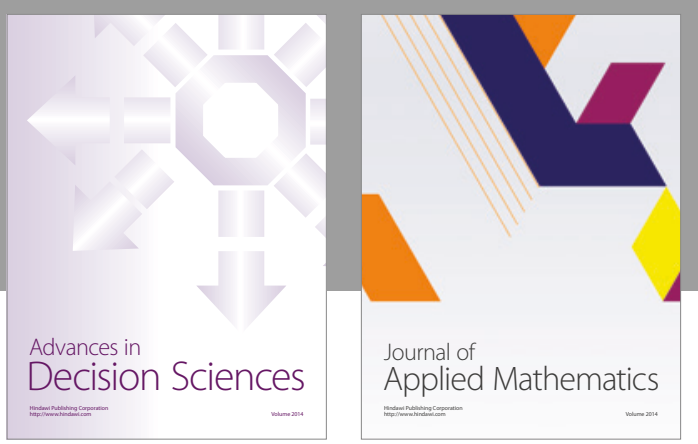

Algebra

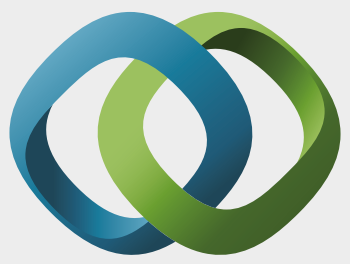

\section{Hindawi}

Submit your manuscripts at

https://www.hindawi.com
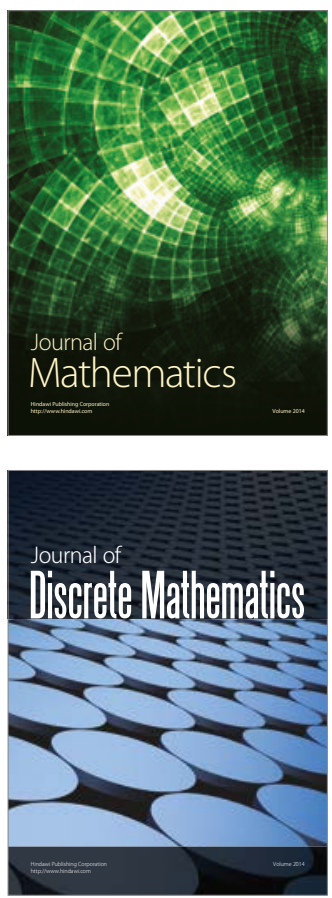

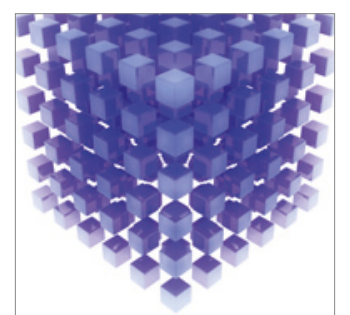

Mathematical Problems in Engineering
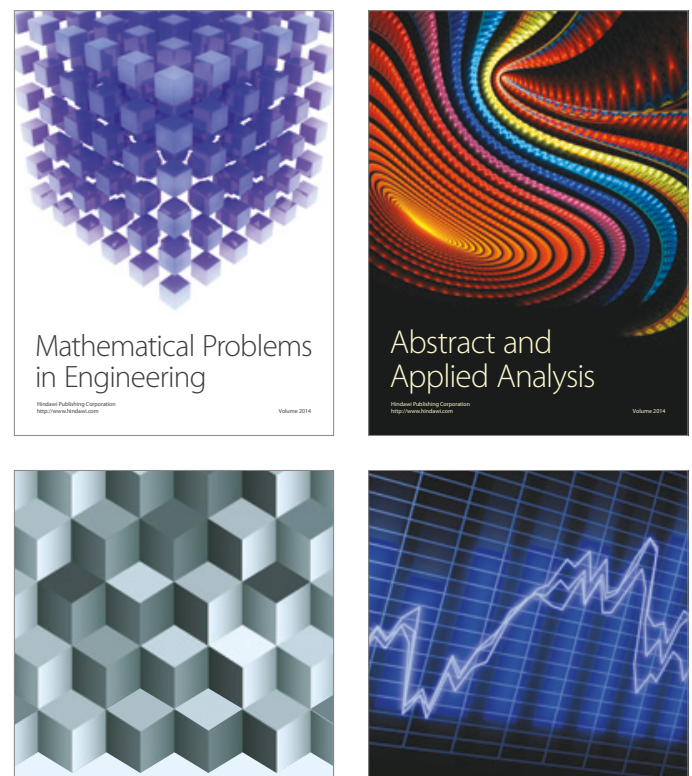

Journal of

Function Spaces

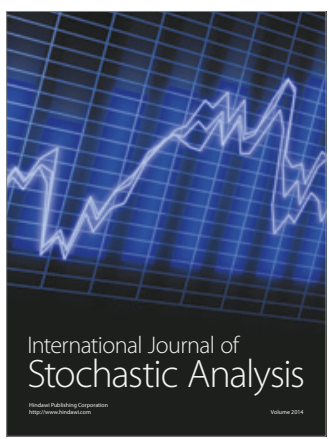

Probability and Statistics
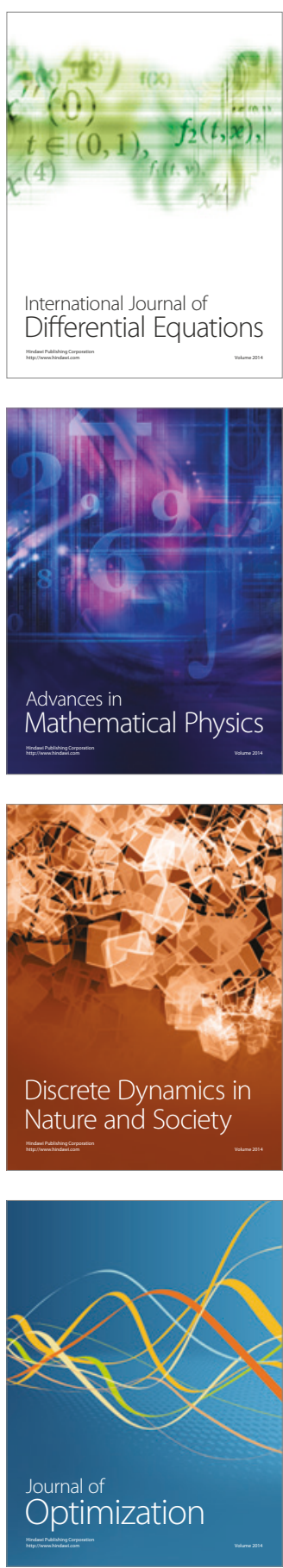